\title{
Plantas acuáticas invasoras presentes en Chile: Distribución, rasgos de vida y potencial invasor
}

\section{Invasive aquatic plants presents in Chile: Distribution, traits of life and invasive potential}

\author{
Jonathan Urrutia ${ }^{1,2^{*}}$, Paulina Sánchez ${ }^{1}$, Aníbal Pauchard ${ }^{1} \&$ Enrique Hauenstein ${ }^{2}$ \\ ${ }^{1}$ Laboratorio de Invasiones Biológicas, Facultad de Ciencias Forestales, Universidad de Concepción, Casilla 160-C, \\ Concepción, Chile. \\ ${ }^{2}$ Escuela de Ciencias Ambientales, Facultad de Recursos Naturales, Universidad Católica de Temuco, Casilla 15-D, Temuco, \\ Chile. \\ *jurrutiaestrada@gmail.com
}

\section{RESUMEN}

\begin{abstract}
Las invasiones biológicas son reconocidas como una de las mayores causas de pérdida de la biodiversidad. Si bien los procesos de invasión se dan tanto en ambientes terrestres como en acuáticos, son los ecosistemas límnicos particularmente sensibles a sus impactos. Dado que la información sobre plantas acuáticas invasoras es escasa en Sudamérica y muy limitada en Chile, el objetivo de este trabajo fue generar información base sobre este grupo de plantas, listando las especies acuáticas invasoras, su distribución, sus rasgos de vida y su potencial invasor (WRA-Ch). Se realizó una revisión exhaustiva en la base de datos Web of Science y otras páginas Web relevantes sobre el comportamiento invasivo de las especies de plantas acuáticas introducidas en Chile. Se encontraron registros para catorce especies de plantas acuáticas invasoras. Myriophyllum aquaticum, Eichhornia crassipes y Egeria densa presentaron el mayor número de referencias, mientras que Limnobium laevigatum y Utricularia gibba mostraron la menor cantidad. La especie con el mayor potencial invasor fue $M$. aquaticum y Veronica beccabunga obtuvo el menor. La Región de Valparaíso presentó la mayor riqueza de plantas acuáticas invasoras, y exhibe además la mayor proporción de dichas especies en relación con el hábitat disponible. El conocimiento de las plantas acuáticas invasoras es fundamental para delinear los aspectos de conservación de los sistemas límnicos de Chile, para así reducir las amenazas sobre estos ecosistemas.
\end{abstract}

Palabras clave: Comportamiento invasivo, impactos, ecosistemas límnicos.

\begin{abstract}
Biological invasions are recognized as one of the major causes of biodiversity loss. Although invasion processes occur in both terrestrial and aquatic environments, freshwater ecosystems are particularly sensitive to invasions impacts. Given that the information on invasive aquatic plants is scarce in South America and highly limited for Chile, the this work aims to generate baseline information about invasive aquatic plants for the country, identifying these species, their distribution, their life-history trades and their invasive potential. A comprehensive search was performed on the Web of Science database and other relevant $W e b$ pages on the invasive behavior of aquatic plant species introduced in Chile. Records for 14 invasive aquatic species were found. Myriophyllum aquaticum, Eichhornia crassipes and Egeria densa showed the highest number of references, while Limnobium laevigatum and Utricularia gibba showed the least. The species with the highest invasive potential was M. aquaticum, while Veronica beccabunga had the lowest. The Region of Valparaiso showed the highest number of invasive aquatic plants, and also exhibits the highest proportion of such species in relation to the available habitat. Knowledge of the invasive aquatic plants is essential to delineate aspects of conservation of Chile fresh water systems, to reduce the threats on these ecosystems.
\end{abstract}

KeYwords: Invasive behavior, impacts, freshwater ecosystems. 


\section{INTRODUCCIÓN}

Las invasiones biológicas son reconocidas como una de las mayores causas de pérdida de la biodiversidad, causando altos impactos sociales y económicos (Pimentel et al. 2005). Las especies invasoras se han convertido en uno de los principales componentes del cambio global (Bradley et al. 2011) y en el caso particular de las plantas invasoras, éstas pueden modificar profundamente los ecosistemas donde se establecen y son además responsables de la homogeneización biótica (McKinney \& Lockwood 1999, Vilà et al. 2011). Además, las plantas invasoras podrían estar acelerando los procesos de extinción de plantas nativas a escala local (Vitousek et al. 1996). Si bien los procesos de invasión se dan tanto en ambientes terrestres como en acuáticos, son los ecosistemas límnicos los más sensibles a nivel mundial (Ricciardi \& MacIsaac 2011). Su vulnerabilidad ha generado una mirada de atención constante, lo que ha permitido revelar patrones y problemas que han mejorado sustancialmente el conocimiento de los efectos de las especies introducidas (Ricciardi \& MacIsaac 2011). En la actualidad los sistemas límnicos se encuentran gravemente amenazados, debido principalmente a la degradación ambiental (urbanización, deforestación, erosión de suelos), esto ha promovido la idea de que son espacios inútiles y que por ende deben ser eliminados (Dudgeon et al. 2006).

La dinámica de los ambientes límnicos (Lampert \& Sommer 1997, Hauer \& Lamberti 2006) brinda una oportunidad para que las plantas acuáticas exóticas se vuelvan invasoras, en este sentido, la disminución de la corriente en años secos o el aquietamiento del flujo de agua en un sistema lótico, genera las condiciones adecuadas para que se produzca la invasión de diferentes especies (Ramírez \& San Martín 2006). Del mismo modo, perturbaciones de carácter antrópico pueden crear condiciones favorables para el establecimiento de nuevas especies (Paine 1966, Fox \& Fox 1986, Pimm 1989, Raffaele 2004). Una perturbación común causada por el hombre es la contaminación por nutrientes, principalmente fosfatos y nitratos, lo que genera un desarrollo exuberante de la flora acuática en general (Ramírez \& San Martín 2006, Chambers et al. 2008). Otro aspecto importante en la invasibilidad de los sistemas acuáticos es el movimiento de propágulos en la superficie del agua, especialmente en arroyos y ríos (Barrat-Segretain 1996). Tal transporte es inherente a estos ambientes y puede servir como mecanismo de dispersión natural para especies invasoras (Ervin et al. 2006). La importancia de los ecosistemas acuáticos en la dispersión de estas plantas ha sido demostrado previamente (Owens et al. 2001, Riis \& Sand-Jensen 2006) y puede alcanzar importantes proporciones si se tiene en cuenta que una porción considerable de especies de plantas acuáticas son capaces de reproducirse asexualmente mediante de la fragmentación de sus tallos (Cook 1990).
En el último tiempo la literatura relacionada a invasiones biológicas ha experimentado un crecimiento considerable a nivel mundial, sin embargo, la información sobre las plantas acuáticas invasoras en Sudamérica y particularmente en Chile sigue siendo escasa. En el contexto sudamericano, destacan los reportes en torno al crecimiento excesivo de Eichhornia azurea y E. crassipes en Brasil (Tundisi et al. 1993), fenómenos de colmatación por plantas acuáticas invasoras en humedales de Colombia (Díaz et al. 2012) y problemas asociados a malezas acuáticas en general en algunas provincias de Argentina (Sabbatini et al. 2014). Para Chile, en tanto, la falta de información es evidente y el único trabajo que toca tangencialmente el tema en cuestión dice relación con malezas que invaden cultivos de arroz (San Martín \& Ramírez 1983). En Chile existe una gran variedad y cantidad de ríos, lagos y humedales, por lo tanto, la invasión de plantas acuáticas podría ser un fenómeno frecuente en el país. Además, gran parte de la población y sus respectivas actividades productivas tienen lugar en las proximidades de los cuerpos de agua (Grantham et al. 2010). Esta situación se traduce en una constante presión de propágulos vegetativos, aumento de las vías de introducción de especies y de la intensidad de las perturbaciones, factores que en última instancia determinan el éxito de una invasión (D'Antonio et al. 1999, Lockwood et al. 2005, Hulme 2007, Hulme et al. 2008, Pysek et al. 2010); y por ende pueden poner en peligro la estabilidad de hábitats tan susceptibles a la invasión como los humedales (Ciotir et al. 2013). Aun cuando Chile tiene numerosos registros de especies de plantas acuáticas invasoras, no existe un catastro sintético de su diversidad y distribución en el territorio nacional. Por lo tanto, el objetivo de este trabajo es generar información base sobre la flora vascular acuática invasora en Chile, al determinar las especies que prosperan en el país, la distribución que presentan, sus rasgos de vida y su potencial invasor.

\section{METODOLOGÍA}

BÚSQUEDA DE INFORMACIÓN

Se elaboró una lista inicial de las plantas vasculares acuáticas introducidas en Chile, considerando principalmente los trabajos de Ramírez \& San Martín (2006) y Fuentes et al. (2013). Para cada especie se buscó información sobre su comportamiento invasivo en sitios $\mathrm{Web}$ oficiales mantenidos por organizaciones reconocidas y que albergan datos relevantes de naturaleza científica o académica. Además, con base en los mismos términos anteriores se realizó una búsqueda en la base de datos de Thomson Reuters (Web of Science, http://thomsonreuters.com/thomson-reutersweb-of-science/), con la siguiente combinación: nombre científico + inva* (e.g. Limnobium laevigatum inva*), esto permitió incluir términos como invasive, invaders o invasion 
que estuvieran incluidos en la descripción. Lo anterior hizo posible obtener una segunda lista de especies para las cuales se registraron antecedentes relacionados con su comportamiento invasivo, países invadidos e impactos sobre los ecosistemas. Además, se buscó información respecto de la taxonomía, origen geográfico, tipo de reproducción, tipo de polinización, tipo de dispersión y año de primer registro para Chile. La clasificación y nomenclatura de las especies fue tomada de Zuloaga et al. (2008) y www.tropicos.org, los rasgos de vida de DiTomaso \& Healy (2003) y Ramírez et al. (1982), y las fechas de primer registro se obtuvieron de Matthei (1995), Fuentes et al. (2013) y de ejemplares depositados en el Herbario de la Universidad de Concepción (CONC).

\section{DisTRIBUCIÓN DE LAS ESPECIES}

La distribución de las especies en Chile se determinó sobre la base de registros del Herbario CONC, en conjunto con los trabajos de Ramírez et al. (1979) y Ramírez et al. (1986), con dichos registros se elaboró un mapa de distribución de las especies.

Con el fin de obtener un diagnóstico de las regiones con una relativa mayor diversidad de plantas acuáticas invasoras, para cada región se calculó un índice de diversidad de especies invasoras corregido por área, dado por la división entre la riqueza específica de plantas acuáticas invasoras y la superficie de hábitat disponible (lagos, lagunas, humedales) expresada en Logaritmo ( $\left.\log _{10}\right)$, y calculada según las bases cartográficas del MIDEPLAN (2006).

\section{AnÁlisis estadístico}

Con el año del primer registro y con el número de regiones invadidas por cada especie, se llevó a cabo una regresión lineal para evaluar la relación entre estas dos variables. Del mismo modo, se realizó una regresión múltiple entre la riqueza de especies de plantas acuáticas invasoras por región como variable dependiente y la superficie de hábitat disponible y la densidad poblacional por región, como variables independientes. Esta última información se obtuvo de www.ine.cl; y las pruebas estadísticas se llevaron a cabo con el software PAST versión 2.17 (Hammer et al. 2001).

\section{ANÁLISIS DE RIESGO (WRA-CH)}

Se calcularon las puntuaciones de la evaluación de riesgo utilizando el protocolo AWRA (Pheloung et al. 1999), adaptado para Chile por Fuentes et al. (2010). Con el fin de obtener una puntuación de evaluación de riesgo se llevó a cabo un análisis en el que se utilizaron catorce especies. El AWRA original contiene 49 preguntas divididas en tres secciones que producen puntuaciones identificables que luego contribuyen a la puntuación total: biogeografía, biología/ecología y rasgos indeseados. Se requiere de un mínimo de diez respuestas por especie para que sea evaluada: dos respuestas en la sección biogeografía, seis en la sección de biología/ecología, y dos en rasgos indeseados. De acuerdo con su puntuación, cada planta invasora se clasificó en alguna de las tres categorías. Las especies que recibieron menos de un punto, que representan un riesgo insignificante de invasión, se clasificaron como de "bajo riesgo". Las especies con un claro potencial de invasión (más de seis puntos) se clasificaron como de "alto riesgo". En el caso de las puntuaciones intermedias, entre 1 y 6 puntos, las especies fueron asignadas a la categoría "riesgo incierto".

\section{RESULTADOS}

De las 26 especies de plantas acuáticas introducidas que crecen en Chile, se obtuvo que catorce (14) tienen un comportamiento invasor en otras partes del mundo (Tabla 1). Estas especies se distribuyen taxonómicamente en 12 familias, donde destaca Hydrocharitaceae con tres representantes. Las especies de las que se obtuvo el mayor número de referencias fueron Myriophyllum aquaticum, Eichhornia crassipes y Egeria densa, por el contrario, las que presentaron menos referencias fueron Limnobium laevigatum y Utricularia gibba. Entre los impactos más frecuentes causados por estas plantas se encuentran: alterar las condiciones físico-químicas del agua, impedir el crecimiento de otras plantas y dificultar el desarrollo de actividades recreativas. Los países que registran el mayor número de estas especies son Estados Unidos (10), Australia (10) y Sudáfrica (7), los continentes con mayor número de países invadidos son Europa (10), África (8) y Asia (8).

La especie con mayor distribución geográfica en Chile es Myriophyllum aquaticum, presente en todas la regiones del país. Por el contrario, Alternanthera philoxeroides, Pistia stratiotes y Veronica beccabunga son las menos comunes; las dos primeras sólo han sido registradas en la Región de Valparaíso y la última sólo en la Región de Los Lagos (Fig. 1). La mayor riqueza de especies de plantas acuáticas invasoras se presenta en la Región de Valparaíso (13) y la menor en la Región de Magallanes (2). Del mismo modo, el índice de diversidad corregido por área de especies invasoras muestra también que la Región de Valparaíso es la que posee una flora acuática invasora relativamente más diversa, con 8,69 especies/log $\left(\mathrm{km}^{2}\right)$, en tanto que la Región de Magallanes es la relativamente menos diversa con sólo 0,56 especies $/ \log \left(\mathrm{km}^{2}\right)$ (Fig. 2).

Al considerar la procedencia geográfica de las especies domina el origen sudamericano. En los atributos de vida hay una prevalencia de la reproducción mixta, destaca la polinización mediada por insectos, y la hidrocoría es el medio más común utilizado en la dispersión (Tabla 1). El WRA-Ch arrojó que la especie con el mayor potencial invasor es Myriophyllum aquaticum con un valor de 27, en tanto Veronica beccabunga presentó el menor potencial 
invasor con un valor de 10. Sin embargo, se debe señalar que todas las especies evaluadas caben en la categoría alto riesgo, ya que poseen un alto valor (Tabla 1).

Por último, la regresión lineal señala que el número de regiones invadidas aumenta cuando la especie presenta un mayor tiempo de residencia (Fig. 3). La regresión múltiple indica que de las dos variables independientes, densidad de habitantes y superficie de hábitat disponible en la región, sólo la primera estaría explicando la riqueza de plantas acuáticas invasoras por región $(\mathrm{p}=0,002)$.

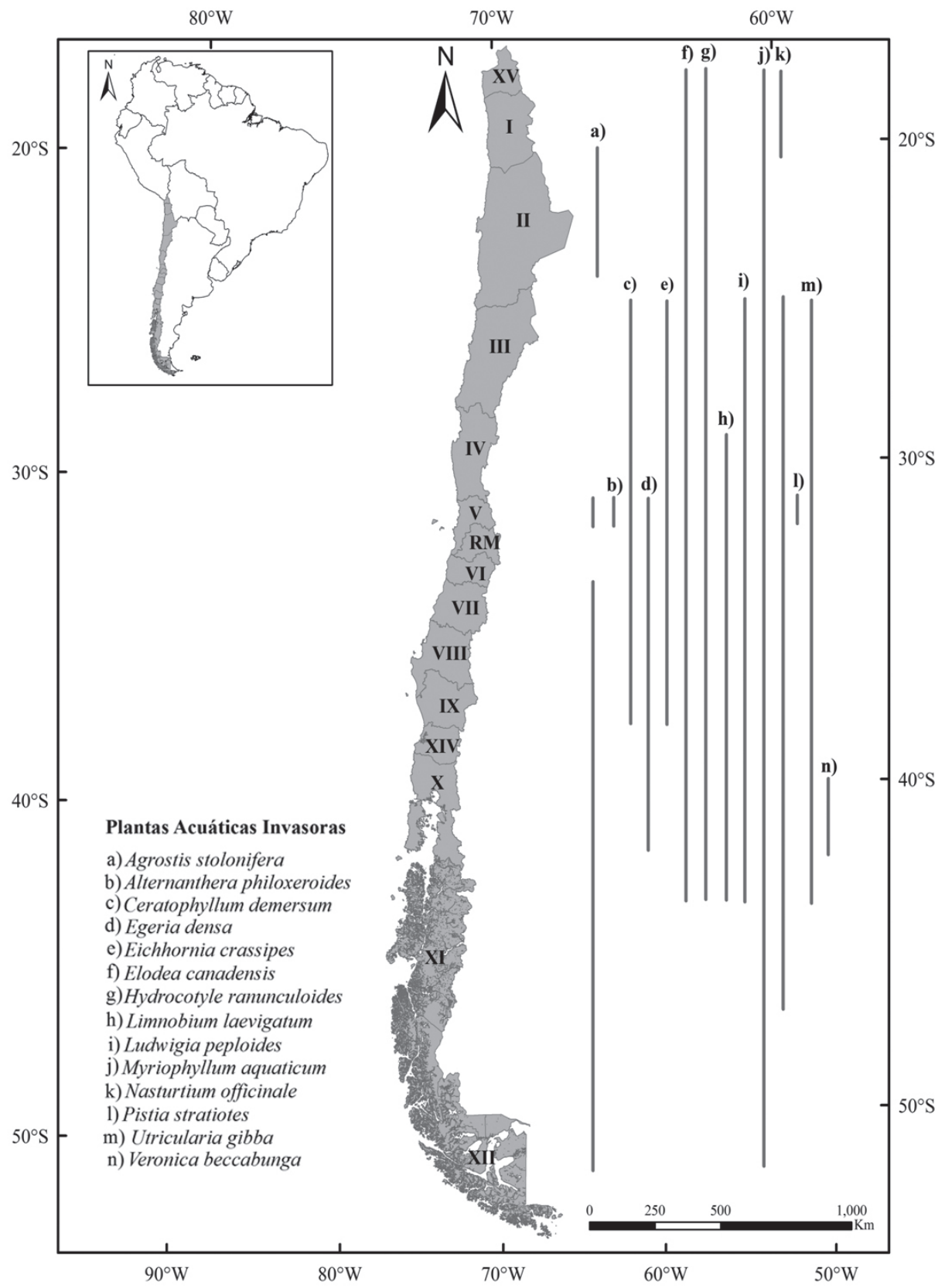

Figura 1. Rangos de distribución de las plantas acuáticas invasoras presentes en Chile. / Distribution ranges of invasive aquatic plants present in Chile. 
Plantas acuáticas invasoras en Chile: URRUTIA, J. ET AL.

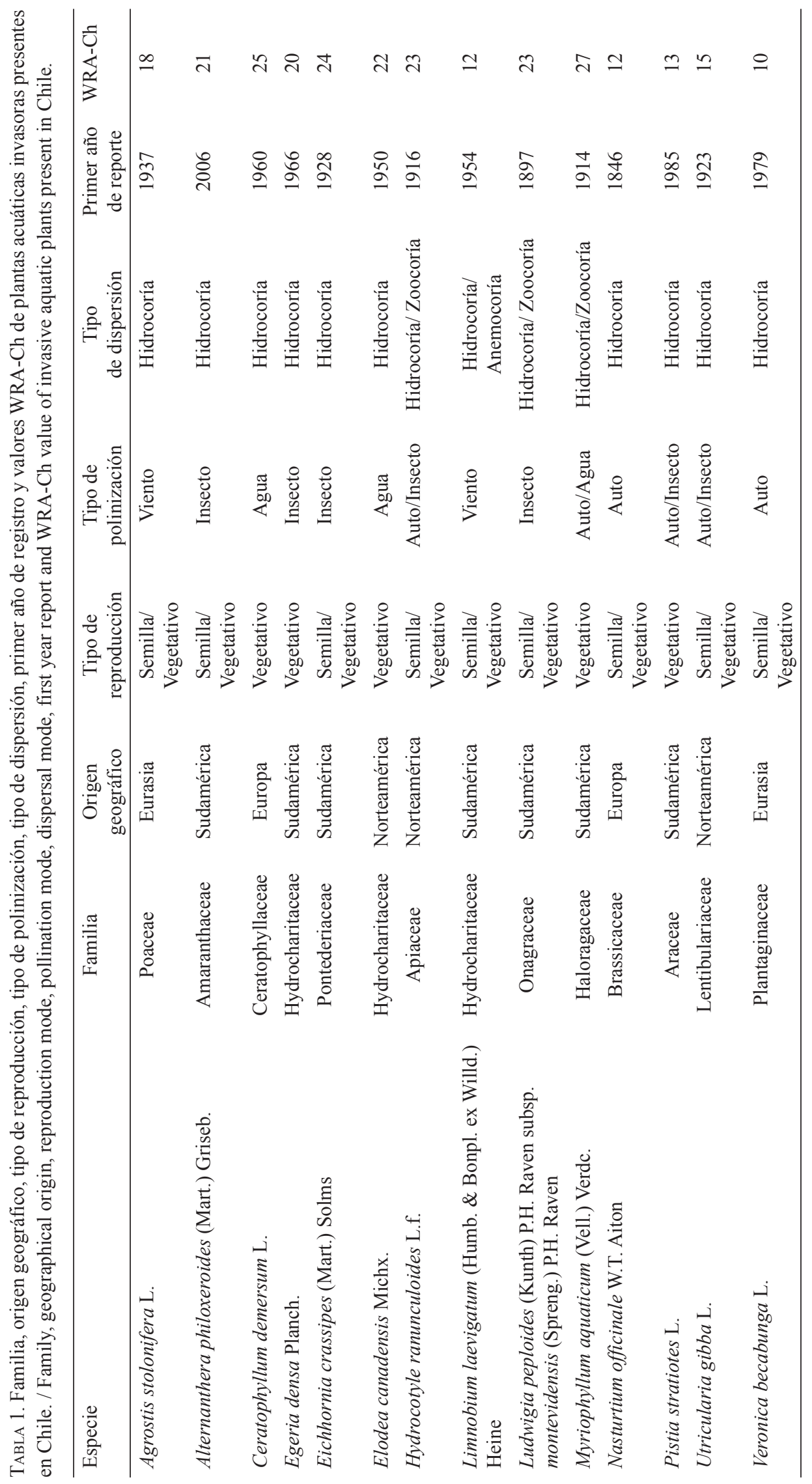


Gayana Bot. 74(1), 2017
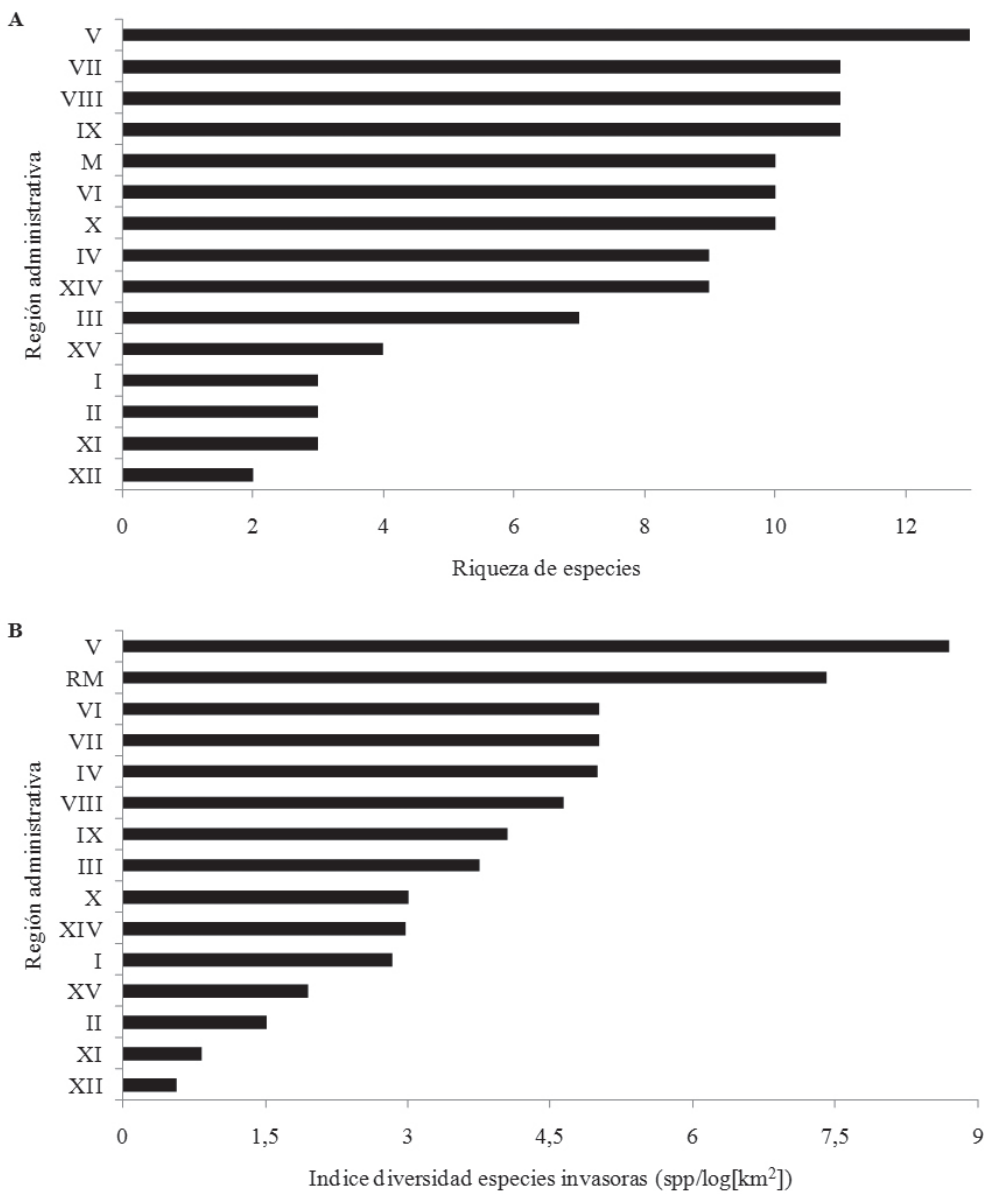

Figura 2. Riqueza de especies por región administrativa (A) y proporción entre la riqueza de plantas acuáticas invasoras y superficie corregida de hábitat disponible en cada región (B). / Species richness by administrative region (A) and proportion between richness of invasive aquatic plants and corrected surface of available habitat for each region (B).

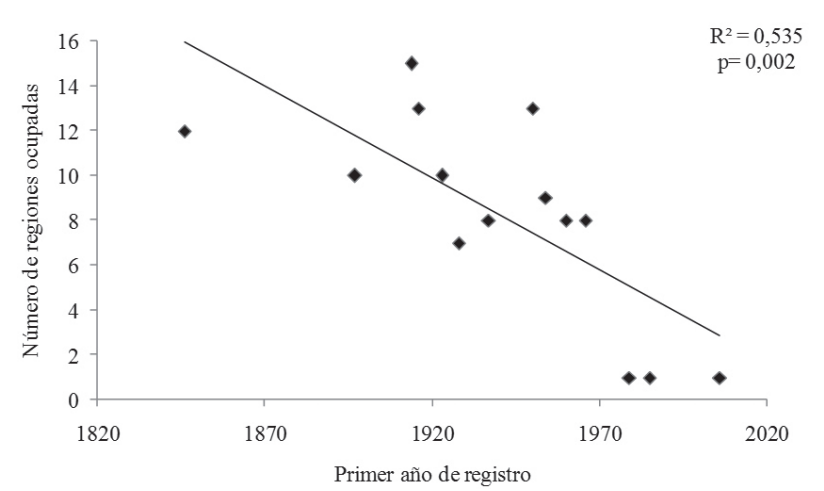

FiguRA 3. Regresión lineal entre el año de primer registro y el número de regiones ocupadas por las plantas acuáticas invasoras. / Linear regression between first year report and the number of regions occupied by invasive aquatic plants.

\section{DISCUSIÓN}

El estudio de las invasiones biológicas en Chile es bastante reciente y la mayor atención se ha centrado en especies de plantas terrestres (Fuentes-Ramírez et al. 2010, Langdon et al. 2010, García et al. 2012, Urrutia et al. 2013). El medio acuático en tanto, sólo en el último tiempo ha capturado el interés de la comunidad científica, lo cual se debe principalmente a la aparición de Didymosphenia geminata (Lyngbye) Schmidt en ecosistemas de agua dulce del sur de Chile (Reid et al. 2012, Rivera et al. 2013). Si bien esta situación ha permitido conocer el proceso de invasión de dicha diatomea (Montecino et al. 2014, Jaramillo et al. 2015, Beamud et al. 2016), la información relacionada a plantas vasculares acuáticas invasoras es aún escasa y dispersa, y está remitida principalmente a colecciones de herbarios y catálogos florísticos locales. 
Hydrocharitaceae, aun cuando mundialmente muestran preferencia por ambientes tropicales (Crow 1993), es la familia con mayor representación de especies en este estudio. Algo similar ocurre en los países europeos, donde la flora introducida de plantas acuáticas es dominada por la misma familia (Hussner 2012). Lo anterior da cuenta de la distribución mundial que exhibe este taxón, ocupando tanto ambientes de agua dulce como salada (Cook 1990), hace sentido con las variadas formas de polinización que exhiben sus miembros y con la preferencia por la propagación vegetativa (Cook \& Urmi-König 1984, 1985).

La única especie presente en todas las regiones del país es Myriophyllum aquaticum (Haloragaceae), lo que puede ser consecuencia de su tipo de reproducción, la cual es casi exclusivamente vegetativa (Xie et al. 2013); una vez que sus tallos se fragmentan, tienen la capacidad de dispersarse a grandes distancias a través de los cuerpos de agua (Wersal \& Madsen 2011), demostrando gran habilidad de colonización y regeneración cuando las raíces se adhieren a los sedimentos (Xie et al. 2013). Todo lo anterior puede estar en relación con el mejor desempeño de los representantes de la familia Haloragaceae en ambientes templados (Crow 1993).

A pesar del fuerte contacto inicial entre Europa y América (Columbian Exchange; Crosby 1972), la mayor parte de las plantas acuáticas invasoras en Chile tienen un origen sudamericano. Esto podría explicarse por la mayor movilidad de propágulos dentro de una misma área geográfica, situación en la cual la dispersión mediada por las personas tiende a ser el principal vector (Murphy et al. 2003, Feldmann \& Nõges 2007). El factor climático no sería tan determinante como en ambientes terrestres, ya que gran parte de la flora dulceacuícola nacional $(70 \%)$ puede considerarse cosmopolita, y por ende ocupa ampliamente el territorio chileno (Ramírez \& San Martín 2008).

Algunos rasgos de vida que poseen estas especies les confieren importantes ventajas competitivas. En este sentido destacan las variadas formas de polinización que presentan y la reproducción asexual como una estrategia de colonización muy útil, más aun cuando todas sus partes vegetativas son susceptibles de ser dispersadas por agua (Johansson \& Nilsson 1993), pudiendo incluso flotar por varios días o semanas, lo que les brinda la posibilidad de ser transportadas a largas distancias (Barrat-Segretain 1996). La hidrocoría como mecanismo de dispersión asoma como el más usado por estas plantas y su importancia ha sido demostrada en varios estudios previos (Staniforth \& Cavers 1976, Rabinowitz 1978, Keddy 1982, Schneider \& Sharitz 1988, Skoglund 1989, Nilsson et al. 1991, Pyšek \& Prach 1993).

Según Ramírez \& San Martín (2006), en Chile la mayor riqueza total de especies de plantas acuáticas (nativas e introducidas) ocurre a los $40^{\circ} \mathrm{S}$, en la Región de Los Ríos, mientras que la situación contraria se da a los $25^{\circ} \mathrm{S}$ en la
Región de Antofagasta. Sin embargo, al considerar sólo las especies introducidas y más específicamente las invasoras (este estudio), se produce un resultado diferente, ya que la mayor riqueza de especies se registra en la Región de Valparaíso. Este patrón se mantiene con los resultados del índice, donde se pondera el número de especies de plantas acuáticas invasoras por la superficie de hábitat disponible, ya que nuevamente es la Región de Valparaíso la que aparece como la más invadida. Esto concuerda con la relación significativa observada, donde se muestra que a mayor densidad poblacional hay una mayor cantidad de especies de plantas acuáticas invasoras, puesto que la Región de Valparaíso es la segunda a nivel nacional con mayor densidad poblacional del país (INE 2012). Sin duda, es el ser humano y su infraestructura asociada (e.g. embarcaciones, canales, desagües), el principal vector en la dispersión de este grupo (Richardson \& Pyšek 2006). Además, la diversidad de plantas acuáticas no sólo responde a factores geográficos, sino también a la presión que provocan las ciudades sobre los ecosistemas acuáticos (Murphy et al. 2003, Feldmann \& Nõges 2007, Chambers et al. 2008). Del mismo modo, la densidad de población humana determina de forma directa el resultado de una invasión, mediante la presión de propágulos, las vías de introducción y la eutroficación, cuando se analiza en conjunto con factores medioambientales (Pyšek et al. 2010). En el caso de las plantas acuáticas existen introducciones accidentales como contaminantes y también intencionales, fundamentalmente como especies ornamentales (June-Wells et al. 2012). Como ejemplo de lo anterior se puede mencionar a Veronica anagallis-aquatica como especie contaminante de semillas (Fuentes et al. 2014) y Eichhornia crassipes, que llegó a Chile con fines ornamentales (Matthei 1995).

La relación entre el primer registro de una especie en el país con el número de regiones invadidas tiene sentido, ya que las plantas introducidas que llevan largo tiempo de residencia en una determinada región tienden a presentar distribuciones más amplias (Rejmánek et al. 2005, Wilson et al. 2007, Küster et al. 2008). El éxito de una invasión puede aumentar si el área invadida posee hábitats similares a aquellos en los que las especies introducidas han evolucionado en sus rangos nativos (Facon et al. 2006). Un problema al trabajar con este tipo de datos es la falta de información histórica para muchas especies introducidas, por lo que el tiempo de residencia es difícil de cuantificar y debe ser estimado indirectamente, basándose en el primer registro conocido para cada especie. Muchas veces el tiempo de residencia es visto como un factor de confusión, cuyo efecto debe ser removido para evaluar los factores biológicos más relevantes que determinan la invasividad (Pyšek \& Jarošík 2005), sin embargo, influye de manera importante en el éxito de un proceso de invasión (Mack 2000, Von Holle \& Simberloff 2005).

Si bien el índice WRA fue concebido para prevenir la 
entrada de plantas nocivas a un país, también se utiliza para evaluar el potencial invasor de las plantas introducidas en un área (Daehler 2005). Cabe señalar que existen vacíos de información para algunas de las especies evaluadas en este estudio, principalmente en el área de reproducción y rasgos indeseados, aun cuando la mayoría de ellas son reconocidas invasoras en varias partes del mundo y generan grandes impactos. Todas las plantas obtuvieron un puntaje "alto", ya que sólo por el hecho de ser de hábito acuático reciben una puntuación adicional en la evaluación (Gordon \& Gantz 2011). Lo anterior se debe principalmente a los efectos que pueden tener en los ecosistemas que invaden (Gordon et al. 2010) y a las altas probabilidades de convertirse en invasoras, incluso más que algunas especies de plantas terrestres (Gordon et al. 2012).

La presencia de 11 de las 14 especies con potencial invasivo en más de la mitad de las regiones del país, se traduce en una amenaza real para los sistemas límnicos de Chile. Es probable que estas especies ya estén causando algún tipo de impacto sobre los cuerpos de agua que habitan y la poca atención sobre ellas es consecuencia de la falta de interés de la sociedad, provocando que dichas situaciones sólo sean dadas a conocer como casos anecdóticos en periódicos de circulación nacional (Diario El Mercurio 2012, Diario El Sur 2013).

Futuros estudios podrían apuntar a explicar los factores que subyacen a la invasión de especies de plantas acuáticas, más aún, teniendo en cuenta lo difícil y costoso que resulta el control de muchas de estas especies. Existen todavía muchos vacíos de información, es necesario conocer los factores que permiten y restringen su proliferación, los impactos que generan en el ambiente y cuantificar su amenaza; esta información será clave en el desarrollo de lineamientos de manejo que permitan evitar daños irreparables en los sistemas acuáticos chilenos.

\section{AGRADECIMIENTOS}

Estudio financiado parcialmente por los proyectos del Instituto de Ecología y Biodiversidad ICM P05-002 y PFB23. EH agradece al Núcleo de Estudios Ambientales (NEA) de la Universidad Católica de Temuco por su apoyo en el presente estudio. Esta investigación se enmarca en el trabajo del Laboratorio de Invasiones Biológicas (LIB), www.lib. udec.cl.

\section{REFERENCIAS}

BARRAT-SEgretain, M. 1996. Strategies of reproduction, dispersion, and competition in river plants: A review. Vegetatio 123: 13-37.

Beamud, S., Baffico, G., Reid, B., Torres, R., Gonzalez-Polo, M., Pedrozo, F., Diaz, M. 2016. Photosynthetic performance associated with phosphorus availability in mats of Didymosphenia geminata (Bacillariophyceae) from Patagonia (Argentina and Chile). Phycologia 55: 118-125.

Bradley, A., Blumenthal, D., Wilcove, D., Ziska, L. 2011. Predicting plant invasions in an era of global change. Trends in Ecology and Evolution 25: 310-318.

Chambers, P., Lacoul, P., Murphy, K., Thomaz, S. 2008. Global diversity of aquatic macrophytes in freshwater. Hydrobiologia 595: 9-26.

Ciotir, C., Kirk, H., Row, J., Freeland, J. 2013. Intercontinental dispersal of Typha angustifolia and T. latifolia between Europe and North America has implications for Typha invasions. Biological Invasions 15: 1377-1390.

Cook, C. 1990. Aquatic plant book. SPB Academic Publishing, The Hague, Netherlands. 228 pp.

Cook, C., Urmi-König, K. 1984. A revision of the genus Egeria (Hydrocharitaceae). Aquatic Botany 19: 73-96.

Cook, C., Urmi-KöNIG, K. 1985. A revision of the genus Elodea (Hydrocharitaceae). Aquatic Botany 21: 111-156.

Crosby, A. 1972. The Columbian Exchange. Biological and cultural consequences of 1492. Greenwood Publishing Company, Connecticut, USA. 268 pp.

Crow, G. 1993. Species diversity in aquatic angiosperms: latitudinal patterns. Aquatic Botany 44: 229-258.

DAeHLer, C. 2005. Upper-montane plant invasions in the Hawaiian Islands: Patterns and opportunities. Perspectives in Plant Ecology, Evolution and Systematics 7:203-216.

D’Antonio, C., Dudley, T., Mack, M. 1999. Disturbance and biological invasions: Direct effects and feedbacks. In: WALKER, L. (ed.), Ecosystems of the world: Ecosystems of disturbed ground, pp. 413-452. Elsevier, Amsterdam, Netherlands.

Diario El Mercurio. URL: http://www.mer.cl. Visto: sábado 29 de septiembre de 2012. Una plaga cubre la Laguna Sausalito.

Diario El Sur. URL: http://www.elsur.cl. Visto: domingo 14 de abril de 2013. La planta invasora "luchecillo". El silencioso enemigo que ataca al lago Lanalhue.

DíAz, A., DíAz, J., VARGas, O. 2012. Catálogo de plantas invasoras de los humedales de Bogotá. Grupo de Restauración Ecológica de la Universidad Nacional de Colombia y Secretaría Distrital de Ambiente, Bogotá D.C., Colombia. $248 \mathrm{pp}$.

DiTomaso, J., Healy, E. 2003. Aquatic and riparian weeds of the west. University of California, California, USA. 442 pp.

Dudgeon, D., Arthington, A., Gessner, M., Kawabata, Z., Knowler, D., Lévêque, C., Naiman, R., Prieur-Richard, A., Soto, D., Stiassny, M., Sullivan, C. 2006. Freshwater biodiversity: Importance, threats, status and conservation challenges. Biological Reviews 81: 163-182.

Ervin, G., Smothers, M., Holly, C., Anderson, C., Linville, J. 2006. Relative importance of wetland type versus anthropogenic activities in determining site invasibility. Biological Invasions 8: 1425-1432.

Facon, B., Genton, B., Shykoff, J., Jarne, P., Estoup, A., DAVID, P. 2006. A general co-evolutionary framework for understanding bioinvasions. Trends in Ecology and Evolution 21: 130-135.

Feldmann, T., NõGes, P. 2007. Factors controlling macrophyte distribution in large, shallow Lake Võrtsjärv. Aquatic Botany 87: 15-21. 
Fox, M., Fox, B. 1986. The susceptibility of natural communities to invasion. In: Groves, R., Burdon, J. (eds.), Ecology of biological invasions: An Australian perspective, pp. 57-66. Australian Academy of Science, Canberra, Australia.

Fuentes, N., Ugarte, E., Kühn, I., Klotz, S. 2010. Alien plants in southern South America. A framework for evaluation and management of mutual risk of invasion between Chile and Argentina. Biological Invasions 12: 3227-3236.

Fuentes, N., Pauchard, A., SÁnchez, P., Esquivel, J., Marticorena, A. 2013. A new comprehensive database of alien plant species in Chile based on herbarium records. Biological Invasions 15: 847-858.

Fuentes, N., Sánchez, P., Pauchard, A., Urrutia, J., Cavieres, L., Marticorena, A. 2014. Plantas invasoras del centro-sur de Chile: Una guía de campo. Laboratorio de Invasiones Biológicas, Universidad de Concepción, Concepción, Chile. 280 pp.

Fuentes-Ramírez, A., Pauchard, A., Marticorena, A., Sánchez, P. 2010. Relación entre la invasión de Acacia dealbata Link (Fabaceae: Mimosoideae) y la riqueza de especies vegetales en el centro-sur de Chile. Gayana Botánica 67: 188-197.

García, R., Fuentes-Ramírez, A., Pauchard, A. 2012. Efecto de dos especies de plantas invasoras fijadoras de nitrógeno sobre las propiedades químicas del suelo en el centro-sur de Chile. Gayana Botánica 69: 189-192.

Gordon, D., Gantz, C. 2011. Risk assessment for invasiveness differs for aquatic and terrestrial plant species. Biological Invasions 13: 1829-1842.

Gordon, D., Gantz, C., Jerde, C., Chadderton, W., Keller, R., Champion, P. 2012. Weed risk assessment for aquatic plants: Modification of a New Zealand system for the United States. PLoS ONE 7(7): e40031. doi:10.1371/ journal.pone.0040031.

Gordon, D., Mitterdorfer, B., Pheloung, P., Ansari, S., Buddenhagen, C., Chimera, C., Daehler, C., Dawson, W., Denslow, J., LaRosa, A., Nishida, T., Onderdonk, D., Panetta, F., Pyšek, P., Randall, R., Richardson, D., Tshidada, N., Virtue, J., Williams, P. 2010. Guidance for addressing the Australian weed risk assessment questions. Plant Protection Quarterly 25: 56-74.

Grantham, T., Merenlender, A., Resh, V. 2010. Climatic influences and anthropogenic stressors: an integrated framework for streamflow management in Mediterraneanclimate California, USA. Freshwater Biology 55: 188-204.

Hammer, Ø., Harper, D., Ryan, P. 2001. Past: Paleontological statistics software package for education and data analysis. Paleontología Electrónica 4: 1-9.

Hauer, F., Lamberti, G. 2006. Methods in stream ecology. Elsevier, Amsterdam, Netherlands. 877 pp.

Hulme, P. 2007. Biological invasions in Europe: Drivers, pressures, states, impacts and responses. In: Hester, R., Harrison, R. (eds.), Biodiversity under threat, pp. 56-80. Royal Society of Chemistry, Cambridge, England.

Hulme, P., Bacher, S., Kenis, M., Klotz, S., Kühn, I., Minchin, D., Nentwig, W., Olenin, S., Panov, V., Pergl, J., Pyšek, P., Roques, A., Sol, D., Solarz, W., Vilà, M. 2008. Grasping at the routes of biological invasions: A framework for integrating pathways into policy. Journal of Applied Ecology 45: 403-414.
Hussner, A. 2012. Alien aquatic plant species in European countries. Weed Research 52: 297-306.

Jaramillo, A., Osman, D., Caputo, L., Cardenas, L. 2015. Molecular evidence of a Didymosphenia geminata (Bacillariophyceae) invasion in Chilean freshwater systems. Harmful Algae 49: 117-123.

JohAnsson, M., NiLsson, C. 1993. Hydrochory, population dynamics and distribution of the clonal aquatic plant Ranunculus lingua. Journal of Ecology 81: 81-91.

June-Wells, M., Vossbrinck, C., Gibbons, J., \& Bugbee, G. 2012. The aquarium trade: A potential risk for nonnative plant introductions in Connecticut, USA. Lake and Reservoir Management 28: 200-205.

Keddy, P. 1982. Population ecology on an environmental gradient: Cakile edentula on a sand dune. Oecologia 52: 348-355.

Küster, E., Kühn, I., Bruelheide, H., Klotz, S. 2008. Trait interactions help explain plant invasion success in the German flora. Journal of Ecology 96: 860-868.

LAmpert, T., Sommer, U. 1997. Limnoecology: The ecology of lakes and streams. Oxford University Press, New York, USA. 324 pp.

Langdon, B., Pauchard, A., Aguayo, M. 2010. Pinus contorta invasion in the Chilean patagonia: Local patterns in a global context. Biological Invasions 12: 3961-3971.

Lockwood, J., Cassey, P., Blackburn, T. 2005. The role of propagule pressure in explaining species invasions. Trends in Ecology and Evolution 20: 223-228.

MACK, R. 2000. Cultivation fosters plant naturalization by reducing environmental stochasticity. Biological Invasions 2: 111122.

Matthei, O. 1995. Manual de malezas que crecen en Chile. Alfabeta Impresores, Santiago, Chile. 545 pp.

McKinney, M., Lockwood, J. 1999. Biotic homogenization: a few winners replacing many losers in the next mass extinction. Trends in Ecology \& Evolution 14: 450-453.

MIDEPLAN. 2006. Ministerio de Desarrollo y Cooperación (MIDEPLAN). Fuentes cartográficas división político administrativa, regiones de Chile.

Montecino, V., Molina, X., Kumar, S., Castillo, M., Bustamante, R. 2014. Niche dynamics and potential geographic distribution of Didymosphenia geminata (Lyngbye) M. Schmidt, an invasive freshwater diatom in Southern Chile. Aquatic Invasions 9: 507-519.

Murphy, K., Dickinson, G., Thomaz, S., Bini, L., Dick, K., Greaves, K., Kennedy, M., Livingstone, S., McFerran, H., Milne, J., Oldroyd, J., Wingfield, R. 2003. Aquatic plant communities and predictors of diversity in a subtropical river floodplain: The Upper Rio Paraná, Brazil. Aquatic Botany 77: 257-276.

Nilsson, C., Gardfjell, M., Grelsson, G. 1991. Importance of hydrochory in structuring plant communities along rivers. Canadian Journal of Botany 69: 2631-2633.

Owens, C., Madsen, J., Smart, R., Stewart, R. 2001. Dispersal of native and nonnative aquatic plant species in the San Marcos River, Texas. Journal of Aquatic Plant Management 39: 75-79.

Paine, R. 1966. Food webs complexity and species diversity. American Naturalist 100: 65-75.

Pheloung, P., Williams, P., Halloy, S. 1999. A weed risk assessment model for use as a biosecurity tool evaluating 
plant introductions. Journal of Environmental Management 57: 239-251

Pimentel, D., Zuniga, R., Morrison, D. 2005. Update on the environmental and economic costs associated with alien-invasive species in the United States. Ecological Economics 52: 273-288.

PIMM, S. 1989. Theories of predicting success and impact of introduced species. In: Drake, J., DiCastri, F., Groves, R., Kruger, F., Mooney, H., Rejmanek, M., Williamson, M. (eds.), Biological invasions: A global perspective, pp. 351367. John Wiley \& Sons, New York, USA.

PYŠEK, P., JAROŠíK, V. 2005. Residence time determines the distribution of alien plants. In: INDERJIT, S. (ed.), Invasive plants: Ecological and agricultural aspects, pp. 77-96. BirkhaüserVerlag, Basel, Switzerland.

Pyšek, P., Jarošík, V., Hulme, P., Kühn, I., Wild, J., Arianoutsou, M., Bacher, S., Chiron, F., Didžiulis, V., Essl, F., Genovesi, P., Gherardi, F., Hejda, M., Kark, S., Lambdon, P., Desprez-Loustau, M., Nentwig, W., Pergl, J., Poboljšaj, K., Rabitschi, W., Roques, A., Roy, D., Shirley, S., Solarz, W., Vilà M., Winter, M. 2010. Disentangling the role of environmental and human pressures on biological invasions across Europe. PNAS 107: 12157-12162.

PYŠEK, P., PrACH, K. 1993. Plant invasions and the role of riparian habitats: A comparison of four species alien to central Europe. Journal of Biogeography 20: 413-420.

RABINOwITZ, D. 1978. Early growth of mangrove seedlings in Panama and a hypothesis concerning the relationship of dispersal and zonation. Journal of Biogeography 5: 113133

RAFFAELE, E. 2004. Susceptibility of a patagonian mallín flooded meadow to invasion by exotic species. Biological Invasions 6: $473-481$.

Ramírez, C., Contreras, D., San Martín, J. 1986. Distribución geográfica y formas de vida en hidrófitos chilenos. Publicación Especial Instituto Geográfico Militar de Chile 1: $103-110$

Ramírez, C., Godoy, R., Contreras, D., Stegmaier, E. 1982. Guía de plantas acuáticas y palustres valdivianas. Universidad Austral de Chile, Valdivia, Chile. 64 pp.

Ramírez, C., Romero, M., Riveros, M. 1979. Habit, habitat, origin and geographical distribution of Chilean vascular hydrophytes. Aquatic Botany 7: 241-253.

Ramírez, C., SAn Martín, C. 2006. Diversidad de macrófitos chilenos. En: Vila, I., Veloso, A., Schlatter, R., Ramírez, C. (eds.), Macrófitas y vertebrados de los sistemas límnicos de Chile, pp. 21-61. Editorial Universitaria, Santiago, Chile.

Ramírez, C., San Martín, C. 2008. Flora acuática. En: Saball, P., Arroyo, M., Castilla, J., Estades, C., Ladrón de Guevara, J., Larraín, S., Moreno, C., Rivas, F., Rovira, J., Sánchez, A., Sierralta, L. (eds.), Biodiversidad de Chile. Patrimonio y desafíos, pp. 358-363. Ocho Libros Editores, Santiago, Chile.

Reid, B., Hernández, K., Frangópulos, M., Bauer, G., Lorca, M., Kilroy, C., Spaulding, S. 2012. The invasion of the freshwater diatom Didymosphenia geminata in patagonia: Prospects, strategies, and implications for biosegurity of invasive microorganisms in continental waters. Conservation Letters 5: 432-440.
Rejmánek, M., Richardson, D., Higgins, S., Pitcairn, M., GrotKopp, E. 2005. Ecology of invasive plants: State of the art. In: Mooney, H., Mack, R., McNeely, J., Neville, L., Schei, P., Waage, J. (eds.), Invasive alien species: A new synthesis, pp. 104-161. Island Press, Washington DC, USA.

RICCIARDI, A., MACIsAAC, H. 2011. Impacts of biological invasions on freshwater ecosystems. In: Richardson, D. (ed.), Fifty years of invasion ecology: The legacy of Charles Elton, pp. 211-224. Blackwell Publishing, Chichester, West Sussex, UK.

Richardson, D., PyŠEK, P. 2006. Plant invasions: Merging the concepts of species invasiveness and community invasibility. Progress in Physical Geography 30: 409-431.

RiIS, T., SAND-JENSEN, K. 2006. Dispersal of plant fragments in small streams. Freshwater Biology 51: 274-286.

Rivera, P., Basualto, S., Cruces, F. 2013. Acerca de la diatomea Didymosphenia geminata (Lyngbye) M. Schmidt: su morfología y distribución en Chile. Gayana Botánica 70: 154-158.

Sabbatini, M., Fernández, O., Bezic, C. 2014. Malezas acuáticas. En: Fernández, O., Leguizamón, E., Acciaresi, H. (eds.), Malezas e invasoras de la Argentina, pp. 783799. Editorial de la Universidad Nacional del Sur, Bahía Blanca, Argentina.

SAN Martín, J., Ramírez, C. 1983. Flora de malezas en arrozales en Chile central. Ciencia e Investigación Agraria 10: 207-222.

SchneIder, R., Sharitz, R. 1988. Hydrochory and regeneration in a bald cypress-water tupelo swamp forest. Ecology 69: 1055-1063.

SkogLund, S. 1989. Seed dispersing agents in two regularly flooded river sites. Canadian Journal of Botany 68: 754-760.

Staniforth, R., Cavers, P. 1976. An experimental study of water dispersal in Polygonum spp. Canadian Journal of Botany 54: 2587-2596.

Tundisi, J., Matsumura-Tundisi, T., CaliJuri, M. 1993. Limnology and management of reservoirs in Brazil. In: Straškraba, M., Tundisi, J., Duncan, A. (eds.), Comparative Reservoir Limnology and Water Quality Management, pp. 22-55. Springer, Amsterdam, Netherlands.

Urrutia, J., Pauchard, A., García, R. 2013. Diferencias en la composición vegetal de un bosque de Araucaria araucana (Molina) K. Koch y Nothofagus antarctica (G. Forst.) Oerst. asociadas a un gradiente de invasión de Pinus contorta Douglas ex Loudon. Gayana Botánica 70: 127135.

Vilà, M., Espinar, J., Hejda, M., Hulme, P., Jarosik, V., Maron, J., Pergl, J., Schaffner, U., Sun, Y., Pysek, P. 2011. Ecological impacts of invasive alien plants: A metaanalysis of their effects on species, communities and ecosystems. Ecology Letters 14: 702-708.

Vitousek, P., D’Antonio, C., Loope, L., Westbrooks, R. 1996. Biological invasions as global environmental change. American Scientist 84: 468-478.

Von Holle, B., Simberloff, D. 2005. Ecological resistance to biological invasion overwhelmed by propagule pressure. Ecology 86: 3212-3218.

Wersal, R., MADSEN, J. 2011. Influences of water column nutrient loading on growth characteristics of the invasive aquatic macrophyte Myriophyllum aquaticum (Vell.) Verdc. 
Hydrobiologia 665: 93-105.

Wilson, J., Richardson, D., Rouget, M., Prochesx, S., Amis, M., Henderson, L., Thuiller, W. 2007. Residence time and potential range: Crucial considerations in modelling plant invasions. Diversity and Distributions 13: 11-22.

XIE, D., Yu, D., You, W., XIA, C. 2013. The propagule supply, litter layers and canopy shade in the littoral community influence the establishment and growth of Myriophyllum aquaticum. Biological Invasions 15: 113-123.

Zuloaga, F., Morrone, O., Belgrano, M. 2008. Catálogo de las Plantas Vasculares del Cono Sur (Argentina, sur de Brasil, Chile, Paraguay y Uruguay). Vol. 1. Pteridophyta, Gymnospermae y Monocotyledoneae. Missouri Botanical Garden Press, Saint Louis, USA. 983 pp.

Recibido: 24.05 .2016

Aceptado: 15.05.2017 